\title{
Precursors for resilience in coral communities in a warming climate: a belief network approach
}

\author{
Scott Wooldridge ${ }^{1,2, *}$, Terry Done ${ }^{1,2}$, Ray Berkelmans ${ }^{1,2}$, Roger Jones $^{3}$, Paul Marshall $^{4}$ \\ ${ }^{1}$ Australian Institute of Marine Science, PMB 3, Townsville, Queensland 4810, Australia \\ ${ }^{2}$ CRC Reef Research Centre, PO Box 772, Townsville, Queensland 4810, Australia \\ ${ }^{3}$ CSIRO Atmospheric Research, PMB 1, Aspendale, Victoria 3195, Australia \\ ${ }^{4}$ Great Barrier Marine Park Authority, PO Box 1379, Townsville, Queensland 4810, Australia
}

\begin{abstract}
This paper explores how successful management interventions might benefit coral reefs during the period of climate warming that is expected in coming decades. To aid this task we have developed a prototype decision-support tool, called 'ReefState', which integrates the outcomes of management interventions within a 'belief network' of connected variables that describe future warming, coral damage and coral recovery. In a case study applied to the inshore waters of the central Great Barrier Reef, Australia, our worst case scenarios, like several others, suggest that reefs will become devoid of significant coral cover and associated biodiversity by 2050. Even under more optimistic (low) rates of future warming, the persistence of hard coral dominated reefscapes beyond 2050 will be heavily reliant on 2 things, the ability of corals to increase their upper thermal bleaching limits by $\sim 0.1^{\circ} \mathrm{C}$ per decade, and management that produces local conditions that constrain excessive algal biomass proliferation during inter-disturbance intervals. Despite being perturbed by a global warming process, management of local ecological factors will thus be of critical importance in shaping the future trajectories of coral reef ecosystems.
\end{abstract}

KEY WORDS: Belief network - Decision support · Uncertainty · Coral bleaching • Adaptation · Resilience · Community composition

\section{INTRODUCTION}

Coral reefs are highly valued and iconic ecosystems that are under severe anthropogenic pressures of many types-from climate change to local overexploitation, pollution and physical destruction (e.g. Bellwood et al. 2004). Not surprisingly, the ecological attribute of 'resilience' in reef populations and ecosystem function is increasingly identified as a key goal for management of coral reefs (e.g. Done et al. 1996, Wilkinson 2004). The achievement of this goal relies in part on developing an understanding of: (1) how important system variables are most likely to change in response to current and future environmental stressors, and (2) how policy and management interventions might assist in safeguarding the system against long-term negative changes to its structure and dynamics.
Given the unprecedented rate of increase in sea temperature that is predicted for the coming decades (IPCC 2001), a key challenge for coral reef managers is to identify a range of management responses that will help to counteract increasingly frequent and severe coral bleaching events (Hughes et al. 2003, West \& Salm 2003, Buddemeier et al. 2004). Typical predictions have major coral bleaching and mortality increasing from rare and localized minor events before 1980, to ubiquitous and severe annual events by the middle of the 21st century (Hoegh-Guldberg 1999, Done et al. 2003, Sheppard 2003). The current concern of coral reef scientists is that when such a disturbance regime is superimposed on anthropogenic pressures on coral reef recovery (e.g. sedimentation, nutrient enrichment, overfishing), then rapid decline in coral reef communities is likely, potentially even overturning their stability over geological time scales (Pandolfi 1999). 
The community state changes of interest relate to the propensities for (1) the dominance of algae instead of coral, or vice versa, (2) low benthic biomass of any description, or (3) selective changes in the predominant types of corals, depending on their heat tolerance, and their opportunities and capability to disperse to and settle and grow on, damaged reef sites (Fig. 1). Assigning levels of confidence in favour of particular coral community trajectories is complicated by the need to reconcile complex processes operating at very different spatial and temporal scales, from global climate systems to the birth and death of individuals in local communities. Moreover, in addition to concerns over the accuracy of future climate projections (Kerr 1997, Allen et al. 2000), important ecological response behaviours remain poorly resolved. This includes uncertainty about the dominant drivers of post disturbance variability in recovery trajectories for coral reefs operating under different levels of environmental and ecological degradation, and the potential (if any) for coral and their dinoflagellate symbionts to adjust or adapt to warming conditions. For example, much uncertainty remains as to the relative importance of eutrophication (bottom-up) and overfishing of herbivores (top-down) as facilitators of major shifts in the coral-algae balance after disturbances (McCook 1996, 1999, Diaz-Puildo \& McCook 2003, McClanahan et al. 2003), whilst recent reviews by Hughes et al. (2003) and Hoegh-Guldberg \& Hoegh-Guldberg (2004) confirm the general lack of understanding regarding the potential contribution that adaptation may make in helping corals survive climate change.

\section{Ecological rates and processes as probability distributions}

It is important that we learn to quantify how these system uncertainties affect our confidence in predicting alternate future trajectories. Such transparency is especially important when we endeavour to compare the relative merits of alternative policies and management interventions in terms of achieving desired system outcomes (Clarke et al. 2001, Kinzig \& Starrett
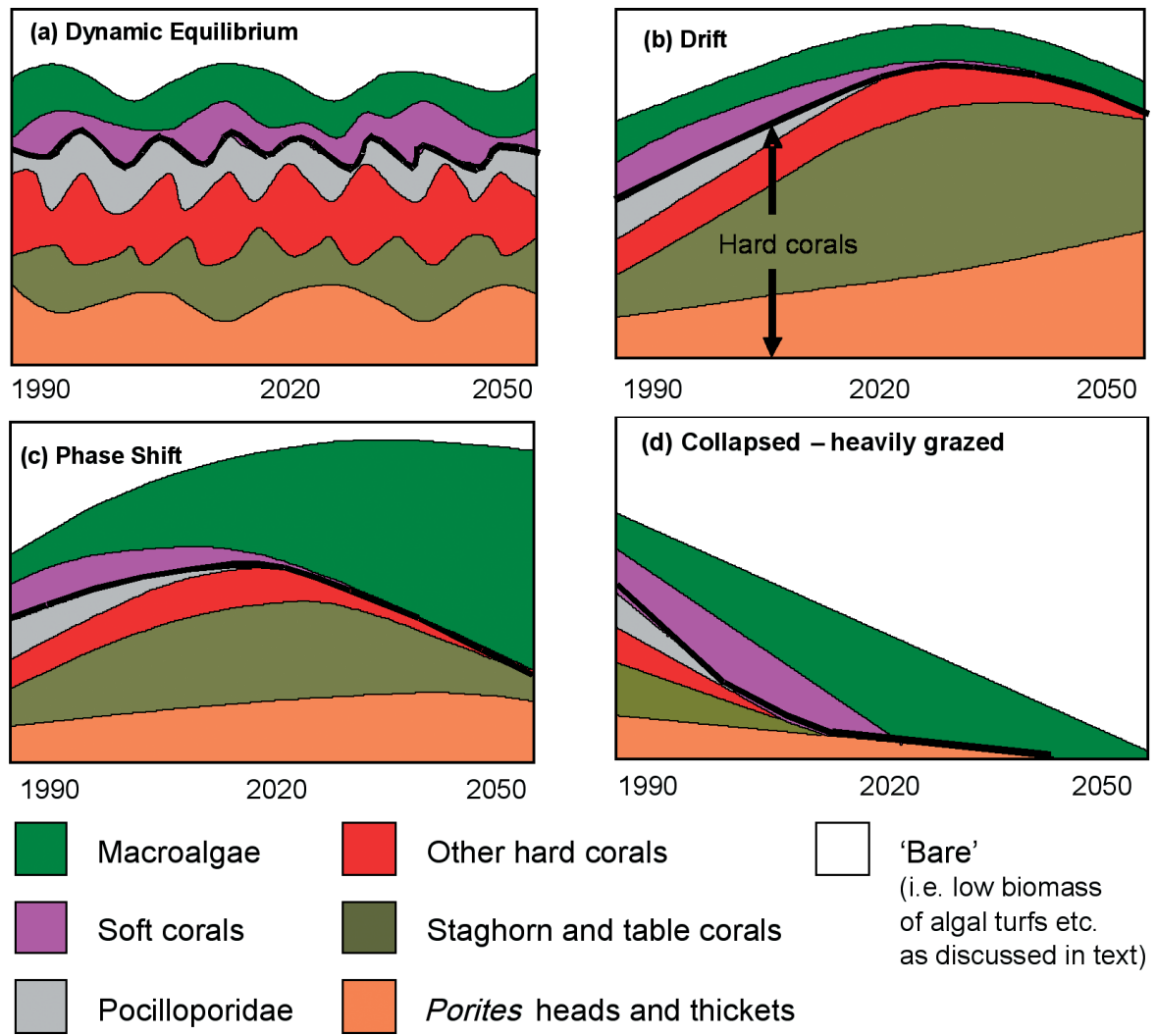

\section{'Bare'}

(i.e. low biomass of algal turfs etc. as discussed in text)

Fig. 1. Possible future scenarios for coral reefs growing under thermal stress (after Done 1999). (a) Physiological tolerance of extant corals with no changes to community structure; (b) 'community-structural drift', the result of differential survival of coral species leading to marked changes in species composition and community structure; (c) 'phase shift' transition to a well-adapted but functionally different group (e.g. macroalgae); (d) 'collapsed-heavily grazed' system dominated by vast areas of bare substrate and rubble 
2003). A useful way of addressing the role of uncertainty is to view the expectation of alternative endpoint responses (e.g. the relative proportions of coral, algae and bare space in an area) with a statistical framework: i.e. we should not expect to be able to predict the behaviour of individual organisms at all locations at all times. Rather, we should focus on the prediction of aggregate statistical properties (e.g. distributions) over relatively large spatial and temporal scales that include realistic measures of prediction uncertainty. For example, we might predict that a single reef has an $80 \%$ chance of being in an algal-dominated state by a certain date, or that 8 out of 10 reefs will be in that state by that time, all else being equal.

It is possible to develop a general statistical framework that both characterises the disturbance and recovery trajectories of an ecological system, and accepts a wide range of information inputs. The latter include empirical evidence, statistical correlations, results from models and expert opinion. In such a framework, if $\mathbf{q}_{t}$ is an $m$-dimensional vector of attributes of observed ecosystem states (e.g. Coral 1, Coral 2, algae, bare space) at time step $t, t=1, \ldots \ldots, \mathrm{n}$, then the modeller's task is to specify a model with structural parameters $\beta$, that accepts inputs $x_{t}$ (e.g. recruitment rate, disturbance regime etc.), and processes them through a transfer function $f($.$) into responses f\left(x_{t}, \beta\right)$. Importantly, also required is $\gamma$ which is a vector of parameters characterizing the errors introduced by uncertainty in the model, the inputs $(x)$, and the response (q).

The vector $\mathbf{q}_{t}$ can be viewed as a random realization from the probability distribution

$$
\mathbf{q}_{t} \leftarrow \phi\left[f\left(x_{t}, \beta\right), \gamma\right], t=1, \ldots \ldots, \mathrm{n}
$$

where $\phi[]$ is a probability distribution conditionally dependent on $f\left(x_{t}, \beta\right)$ and $\gamma$. The role of the error parameter vector $\gamma$ is to guarantee that no more structural explanation remains in the residuals $\left[\mathbf{q}-f\left(x_{t}, \beta\right)\right]$.

\section{Belief networks}

The belief network approach to ecosystem prediction (Reckhow 1999, Borsuk et al. 2004, Wooldridge \& Done 2004) attempts to represent the joint distribution of important systems variables (as described by Eq. 1) with a network (i.e. graphical) model. In the network model, nodes are used to represent important system descriptor and response variables (e.g. climate, local environment, community composition). Links between nodes represent dependency relationships between variables. These relationships may reflect direct causal dependencies or the aggregate effect of more complex associations (Pearl 1999). The range of possible out- comes for a node is expressed by a probability distribution. The propensity for a node to take on a particular value is determined by the node-link dependency structure of the network model, which captures the simple fact that the joint distribution of a collection of random variables can be decomposed into a series of conditional models. For example, consider the belief network model in Fig. 2, which describes the situation in which the degree of future increase in summer sea surface temperatures $(A)$ is conditional on the rate of global warming $(B)$, which in turn is conditional on the levels of greenhouse gas emissions $(C)$ and the sensitivity of the climate to those emissions $(D)$. By using the concept of 'conditional independence' we can write the factorisation of the joint distribution as $\mathrm{p}(A, B, C, D)$ $=\mathrm{p}(A \mid B) \cdot \mathrm{p}(B \mid C, D) \cdot \mathrm{p}(C) \cdot \mathrm{p}(D)$. The resulting series of conditional models capture the notion of modularity (i.e. a complex system built from combining simpler parts) and reflect the fact that some states in our model domain will tend to occur more frequently when other states are also present. The factorisation also demonstrates the quantitative meaning of the links in a belief network, and hence what we need to specify to turn the network (i.e. graphical) structure into a probability distribution. For each node, we need its conditional probability of taking a certain value, given the values of its parents (i.e. the node[s] that are 'up-arrow' from it). In the general case, suppose that the set of variables in a belief network is $\left\{A_{1}, A_{2}, \ldots, A_{n}\right\}$ and that parents $\left(A_{i}\right)$ denotes the set of parents of the node $A_{i}$ in the belief network. Then the joint probability distribution for $\left\{A_{1}, A_{2}, \ldots, A_{n}\right\}$ is

$$
\mathrm{p}\left(A_{1}, \ldots \ldots A_{\mathrm{n}}\right)=\prod_{i=1}^{\mathrm{n}} \mathrm{p}\left(A_{i} \mid \operatorname{parents}\left(A_{i}\right)\right)
$$

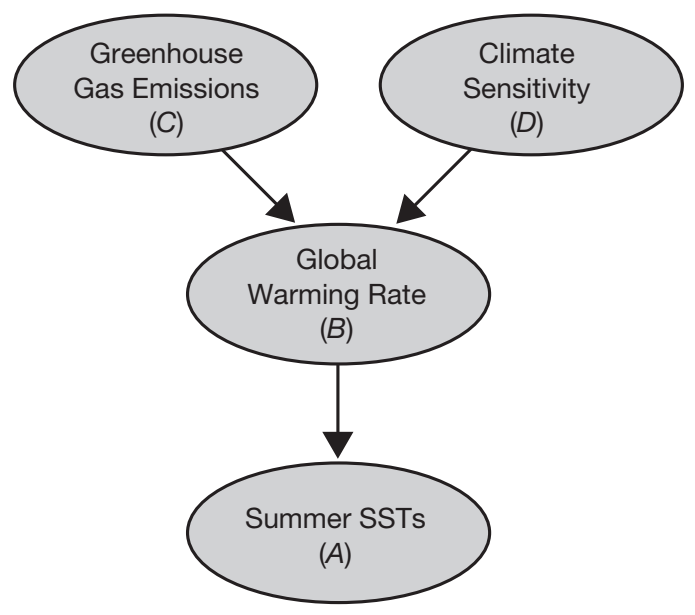

Fig. 2. 'Belief network' representation of causal chain leading to global warming induced changes in summer sea surface temperature (SST). In the network, nodes are used to represent important domain variables, and links between nodes represent dependency relationships 
Belief network modelling is extremely flexible because the conditional probabilities of any link (be it direct causal or aggregate) can be quantified independently. The links may be based on combinations of (1) empirical data, (2) statistical associations derived from historical data, (3) mathematical representations of dominant mechanistic processes, and (4) probabilistic quantities elicited from scientific experts. Any or all are appropriate, as long as the uncertainty associated with each is quantified as a conditional probability distribution.

Importantly, the process of characterising the behaviour of a system in terms of a series of local conditional probabilities in a belief network also produces a framework that correctly propagates system information and associated uncertainties globally (Pearl 1986). This propagation process allows us to infer whether observational evidence at a given node should influence the belief in the occurrence of unobserved events (or system states) at other connected nodes and (perhaps just as importantly) by how much, given the role of uncertainty. (Description of the analytical processes to make such inferences is beyond the scope of this paper but, in brief, they are based on the application of probability calculus and Bayes theorem [Gelman et al. 1997] within the node-link dependency structure of the model domain.)

In this paper, we adopt a generalised belief network modelling approach (Pearl 1988, Neapolitan 1990) to explore some potential precursors for resilience in coral cover and composition during coming decades. Initially, we outline the development of a prototype decision-support tool, called 'ReefState'. ReefState utilises a series of linked belief networks to integrate a purported management outcome (algal biomass) into a continuous causal chain of network variables that describe future warming, coral damage, and coral recovery. Prostrate fleshy types of algae that form dense carpets are especially important in pre-empting space on coral reefs and preventing the establishment of new coral colonies (McCook 1999). ReefState thus integrates the impact of different rates of algal biomass accumulation with a range of plausible global warming scenarios plus different rates of thermal adaptation in corals to investigate precursors for the long-term maintenance of hard coral dominance. These precursors are not only subject to effects of coral reef management (extent of accumulation of algal biomass as determined by water quality, grazing rates and seasonal export of algae), but are also subject to outcomes of international policies (i.e. rate of greenhouse gas emissions as a determinant of rate of global warming).

\section{MATERIALS AND METHODS}

Belief network framework. We developed a model called ReefState (Fig. 3) that utilises the belief network approach to update our strength of belief about what coral reefs may look like over the coming decades. We were particularly interested in identifying system entry points where management efforts may be best directed in order to achieve the desirable outcome of resilience in relation to hard coral cover. As such, we focused on developing a cohesive network description that captured the important aggregate causal relationships that underpin the functional behaviour of coral reef ecosystems, not necessarily the detail of smallscale processes. For example, it is reasonably well understood that the severity of climate induced coral bleaching is regulated by the interplay of a host of complex environmental and physiological processes (Coles \& Brown 2004). However, from a management point of view, we consider a more useful perspective to be the aggregate behavioural response of the coral community as it relates to the magnitude of temperature elevation, and the duration of exposure for any individual warming event (Berkelmans 2002).

The ReefState model components, its overall function, and its use of probability distributions to characterize important ecosystem relationships (and associated uncertainties), are detailed in Appendix 1 (www. int-res.com/journals/suppl/wooldridge_appendix.pdf) and thus summarised only briefly here. At its core, ReefState is a non-spatial reef-scale model that 'grows' percentage cover of corals and macroalgae at different rates and periodically kills some of the corals (but not the macroalgae) by bleaching events caused by summer heatwaves. Each benthic attribute grows according to a space-limiting logistic function that declines to zero as total benthic cover approaches $100 \%$. Competitive growth preference rules for available substrate rank corals as superior competitors to macroalgae, but assume neutrality between different corals, allowing wins and losses to be determined on a random event basis. The frequency and intensity of summer heatwaves are driven by the rate of climate change; the amount of coral that is killed depends on the heatsensitivity of the particular coral species; the sensitivity of the corals is determined by species and rate of adaptation; the intrinsic coral growth rates at all times other than the heatwave are fixed for each species. The expansion of macroalgae to colonise bare substrate (made available following disturbance) can occur at either 'potential' (i.e. unconstrained) or constrained rates. We investigated 'medium' and 'high' growth constraints that reduce the potential recovery period addition in macroalgae cover by 50 and $100 \%$ respectively. The level of algal constraint is assumed to be 
a
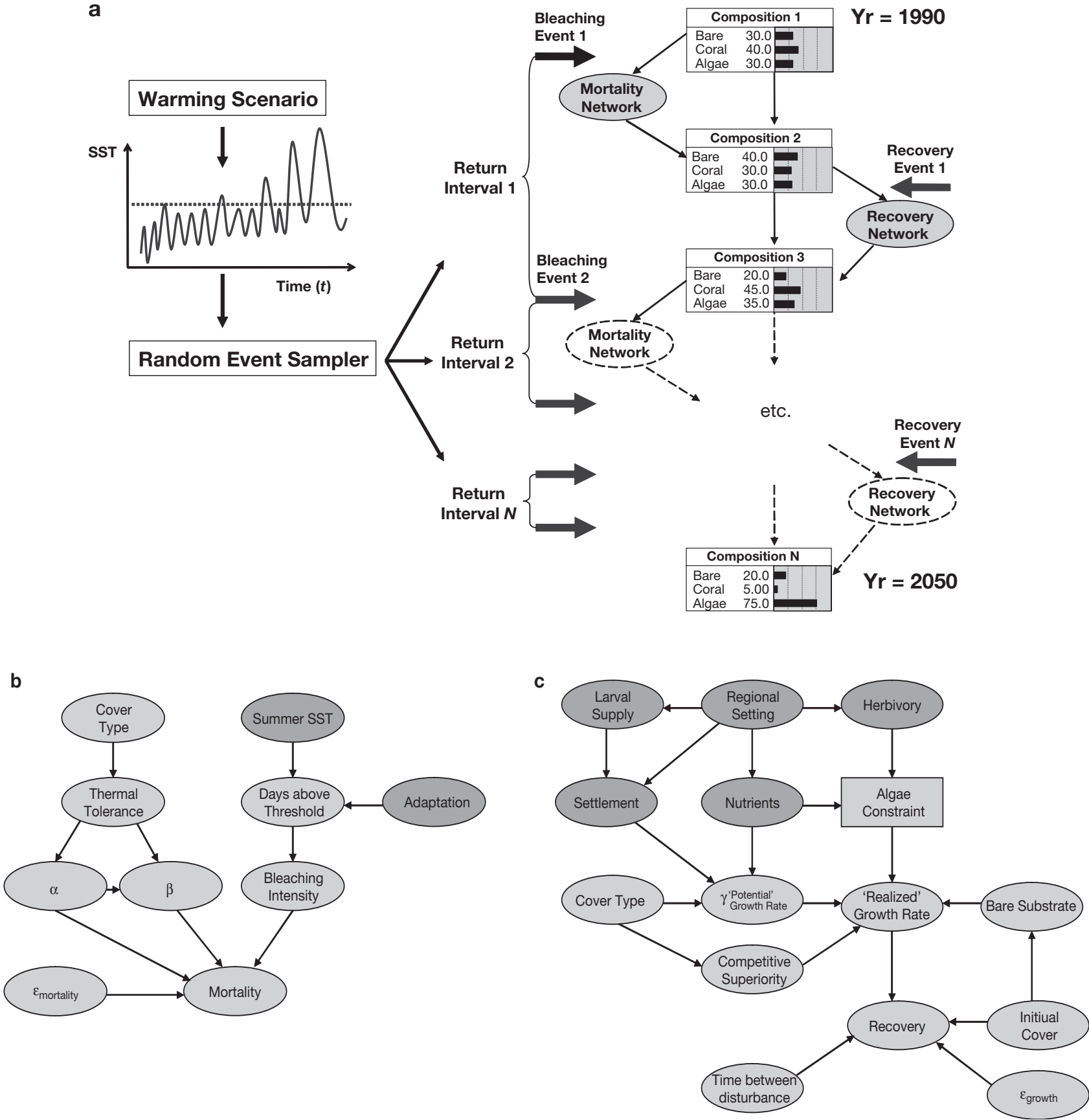

Fig. 3. (a) Schematic representation of 'ReefState' model demonstrating how 'strength of belief' about future coral status (at a particular reef location) is updated based upon expectation for return interval between recurrent bleaching events, mortality profile, and recovery profile; (b) system variables and dependencies used to update expected mortality profile resulting from bleaching events of different intensities; (c) system variables and dependencies used to update expected recovery profile of coral communities between bleaching events

potentially dependent on management interventions that affect water quality and levels of herbivory.

Emergent endpoint responses. We chose coral cover and community composition as the emergent system properties of interest. Corals with branching growth forms, rapid growth rates and thin tissue layers appear to be most sensitive to bleaching, while slow-growing, thick-tissued, massive corals appear less sensitive (Marshall \& Baird 2000, Loya et al. 2001). We therefore chose to model the dynamics of a simplified coral community that consists of (1) a thermally tolerant, slow growing coral (characterised by many Porites species), 
(2) a thermally sensitive, fast growing coral (characterised by many Acropora species), and (3) thermally immune algae (strictly macroalgae such as Dictyota spp. or Lobophora spp., which often form dense carpets across reef substrata). For any space not occupied by any of these 3 benthic types, we use the term 'bare' shorthand. In reality, such spaces are quickly colonised by algal turfs and microbial films. The point is that in this model, it is only 'bare' spaces into which corals and macroalgae can grow.

Within the ReefState model, the propensity for the indicator coral community to assume different compositional states (i.e. coral-algae-bare combinations) over time is driven by the interplay between (1) the return interval characteristics of bleaching events (of a given intensity), and quantified beliefs regarding (2) damage to different community components and (3) the potential for recovery between recurrent bleaching events (Fig. 3a). The return interval characteristics for future bleaching events were modelled as a stochastic process (details in Appendix 1) whose dynamics depend on the future rate of warming in sea surface temperature (SST) and are based on climate projections generated by the Intergovernmental Panel on Climate Change (IPCC 2001).

Mortality and recovery networks. For bleaching events of different intensities (see Table A1: Appendix 1), quantification of the level of mortality expected in the bottom coverage of Porites spp., Acropora spp. and algae is facilitated by the mortality belief network in Fig. 3b. The onset of sub-lethal bleaching is based on location-specific temperature-duration thresholds that are determined by the location's history of thermal acclimatization and adaptation (Berkelmans 2002). We used the number of days of summer SST spent above this threshold as a surrogate measure for the intensity of a bleaching event. The subsequent mortality response arising from a bleaching event of given intensity is conditionally dependent on the differential thermal tolerances assigned to the Acropora spp., Porites spp. and algae (see Fig. A3: Appendix 1).

On return to non-bleaching conditions, the most probable trajectories of the bottom coverage of Porites spp., Acropora spp. and algae is quantified by the recovery belief network in Fig. 3c. The 3 community elements achieve growth rates that are conditionally dependent upon several things: the colony growth rate unconstrained by competitive interactions; the discounting effect on growth due to competitive exclusion; the amount of bare substrate available (i.e. not already occupied by coral or algae); and the external constraints on algae expansion (such as water quality and herbivory). We may think of high levels of 'algae constraint' as the management target to maximise both opportunities for coral recruitment and rates of coral growth and survival.
Linking functions. The conditional probability distributions for the node-link dependency relationships were determined using a functional approach (details in Appendix 1). In summary, the conditional distribution of a nodal variable $X$ is derived using a functional relationship of the form

$$
X=f(p, \theta, \varepsilon)
$$

where $p$ is the set of immediate causes (or parents) of $X, \theta$ is a vector of parameters of the function relating $p$ and $X$, and $\varepsilon$ is an error (or disturbance) term (after Borsuk et al. 2004). In Eq. (3), causal relationships representing physical mechanisms are summarised by mathematical functions and uncertainties are introduced by regarding the arguments of the functions as random variables with user-assigned probability distributions derived through a combination of prior judgement and statistical updating (Gelman et al. 1997). The probability distribution assigned to the 'catch-all' error term $\varepsilon$ represents the effects of exogenous factors that, for reasons of either choice or lack of information, have not been included in the analysis. It is used to correct for any unexplained stochasticity (i.e. uncertainty) that is not captured by the structural element of a functional relation. A common assumption is that $\varepsilon$ is an independent and identically distributed Gaussian random variable with zero mean and specified variance.

Scenario simulation. Execution of the ReefState model to quantify the time-dependent state of the coralalgae-bare indicator community is based on a Monte Carlo simulation approach. The simulation randomly samples $(n=10000)$ damage and recovery sequences between 1990 and 2050 according to decadal encounter probability statistics for bleaching events of a given intensity (Fig. A1: Appendix 1). For each randomised simulation sequence, the trajectory of the coral-algaebare combination is propagated through time based on the most probable outcome from each damage and subsequent recovery event. For example, on the instantiation of a bleaching event of a given intensity, random samples $(n=10000)$ of the parameter and error distributions of the mortality belief network are used to give a deterministic range of damage possibilities. The median (50th percentile) response from these damage possibilities is taken as the most probable damage outcome, and is used to update the pre-bleaching coral-algae-bare combination. In a similar fashion for each 6 mo (or part thereof) period following a bleaching event, random samples $(\mathrm{n}=10000)$ are drawn from the parameter and error distributions of the recovery belief network to give a deterministic range of recovery possibilities. The median response from these recovery possibilities is used to update the post-bleaching coral-algae-bare combination. The explicit parameter covariance relationships are maintained in the sampling process. 
Fig. 4. (a) Central GBR showing approximate bounds of inshore study area (shaded region); (b) projected increases in mean monthly summer sea surface within study area for high and low warming scenarios; (c) temperatureduration bleaching curve for Middle Reef based on 1990 response data, showning projected 2050 threshold assuming an adaptation rate of $0.1^{\circ} \mathrm{C}$ decade $^{-1}$ temperatures, SST (December-March)
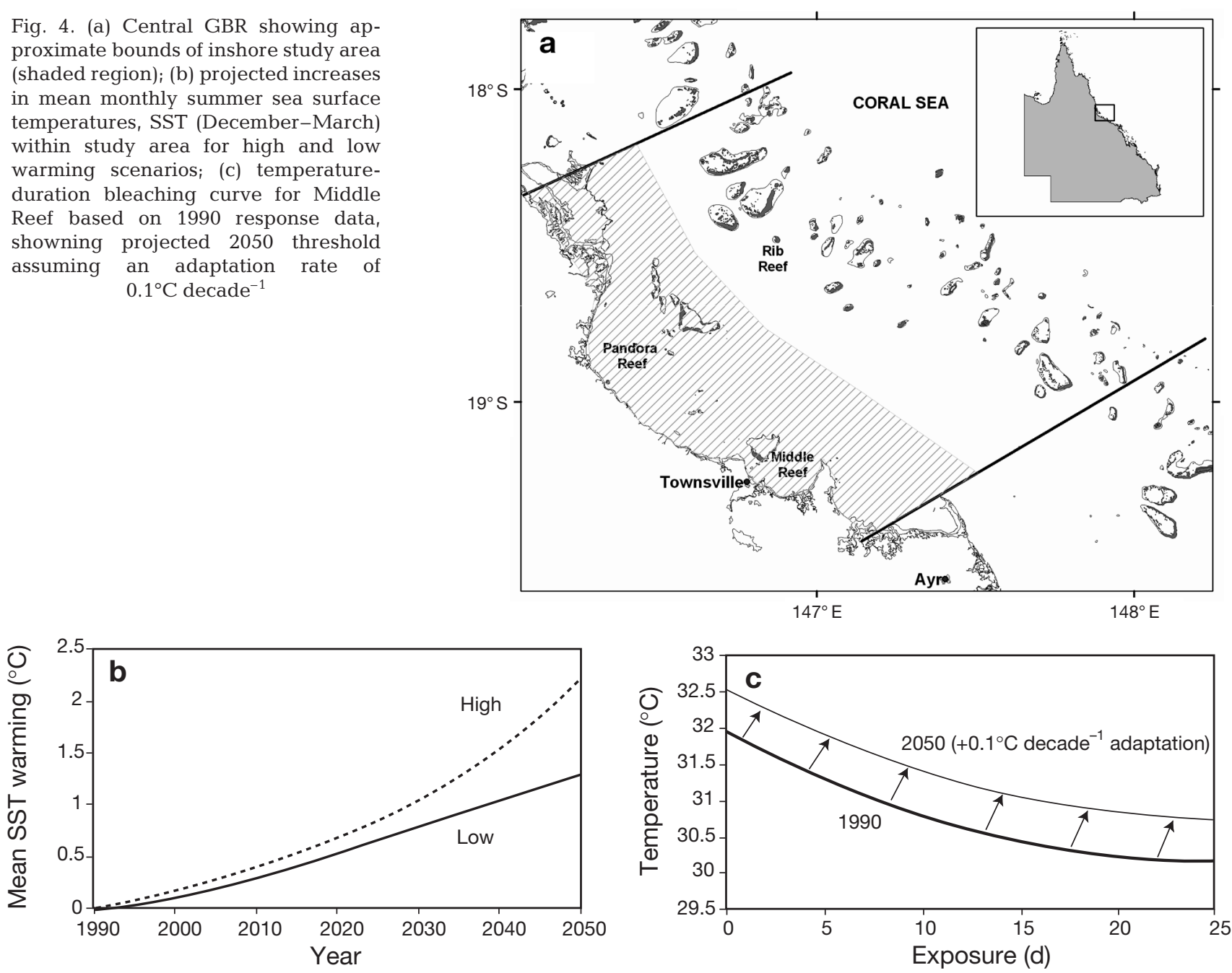

Case study application. We use these tools and approaches to investigate future prospects for inshore reefs of the central Great Barrier Reef (GBR) off Townsville (Fig. 4a). These reefs experienced extensive bleaching in both 1998 and 2002, and are in a recognised 'hotspot' for anomalous summer SSTs (Berkelmans et al. 2004). In addition to the increasing thermal stress within the region, it is widely believed that the capacity for post-disturbance recovery of corals on these inshore reefs is compromised by low herbivore populations and enhanced sediment and nutrient enrichment along gradients of terrestrial runoff (Scott \& Russ 1987, Bell 1992, Brodie \& Furnas 1996, McCook 1996, 1999, Klumpp et al. 1999). In many reefs around the world, abundance of herbivorous fishes is low due to high and indiscriminate fishing pressure. On the GBR, herbivorous fishes are not specifically targeted by commercial or recreational fishers, but we know of no quantitative data about the extent to which their populations are affected through losses to fishing bycatch or by removal of their preda- tors. Their low abundance on some inshore reefs may be due to elevated levels of suspended solids that may provide less suitable habitat for herbivorous fishes (Williams 1991).

We investigated the likelihood of the following 5 outcomes by the year 2050: (1) dominance by corals; (2) dominance by Acropora spp.-like corals; (3) dominance by Porites spp.-like corals; (4) dominance by macroalgae; (5) a bare reef dominated by neither hard corals nor macroalgae. We made these investigations under 2 scenarios for global warming (Fig. 4b), both of which are considered equally plausible by climate scientists (IPCC 2001): the 'low warming' scenario (an increase above a 1990 baseline of 1 to $1.5^{\circ} \mathrm{C}$ by 2050 , and the 'high warming' scenario ( 2 to $2.5^{\circ} \mathrm{C}$ by 2050 ). We set a 'bleaching onset' threshold for the study area based on Berkelmans' (2002) local temperatureduration bleaching curve for the 1990s (Fig. 4c). The intensity of the event and degree of coral mortality were determined by the number of days with temperatures above and to the right of the curve. For our simu- 
lation, we assumed first, that the intensity threshold was fixed, and second that it 'adapted' (moved to the right) at a rate of $+0.1^{\circ} \mathrm{C}$ decade $^{-1}$ (i.e. reflecting increasing heat tolerance in both types of corals at about half the low rate of warming).

To ensure consistency between scenario projections, we began all the simulations with the same initial community structure; total cover being made up of $30 \%$ Acropora spp., $20 \%$ Porites spp., $10 \%$ algae and $40 \%$ bare substrate. This initial community structure broadly approximates the average 1990 inshore reef condition, based on archived data from a number of inshore reefs (see www.aims.gov.au/pages/research/ reef-monitoring/reef-monitoring-index.html).

\section{RESULTS AND DISCUSSION}

\section{Coral to algal transition}

A typical model trajectory under conditions of high warming and unconstrained growth of macroalgae is provided in Fig. 5, including estimates of simulated variability (i.e. uncertainty) in the coral-algae-bare endpoint outcomes. All other things being equal, the more the posterior (after the evidence) distribution of the pre- dicted outcome concentrates its probability mass about a particular point in the ternary space, the more precise (or certain) our belief in that outcome. In this example, despite the major decrease in certainty with time, the strong central trend to an algal dominated reef state is not in doubt. Many alternate trajectories (to numerous to reproduce here) have been simulated with ReefState given different eventualities for (1) future warming, (2) inter-disturbance reef conditions, and (3) thermal adaptive capacities in corals. Our intention here is to summarise the key findings by reporting the effect of specific model selections on the likelihood of occurrence of prescribed endpoint outcomes (i.e. zones of interest in the coral-algae-bare ternary space).

\section{Retention of coral dominance}

To assess the likelihood of hard coral dominated reefscapes up to 2050, we considered the joint probability distribution describing the likelihood of combined Acropora spp. and Porites spp. cover being $>30 \%$ and algae cover being $<20 \%$. Not surprisingly, the nature of this distribution is strongly influenced by the applied level of constraint to algal growth (Fig. 6a,b). For example in the low warming scenario,
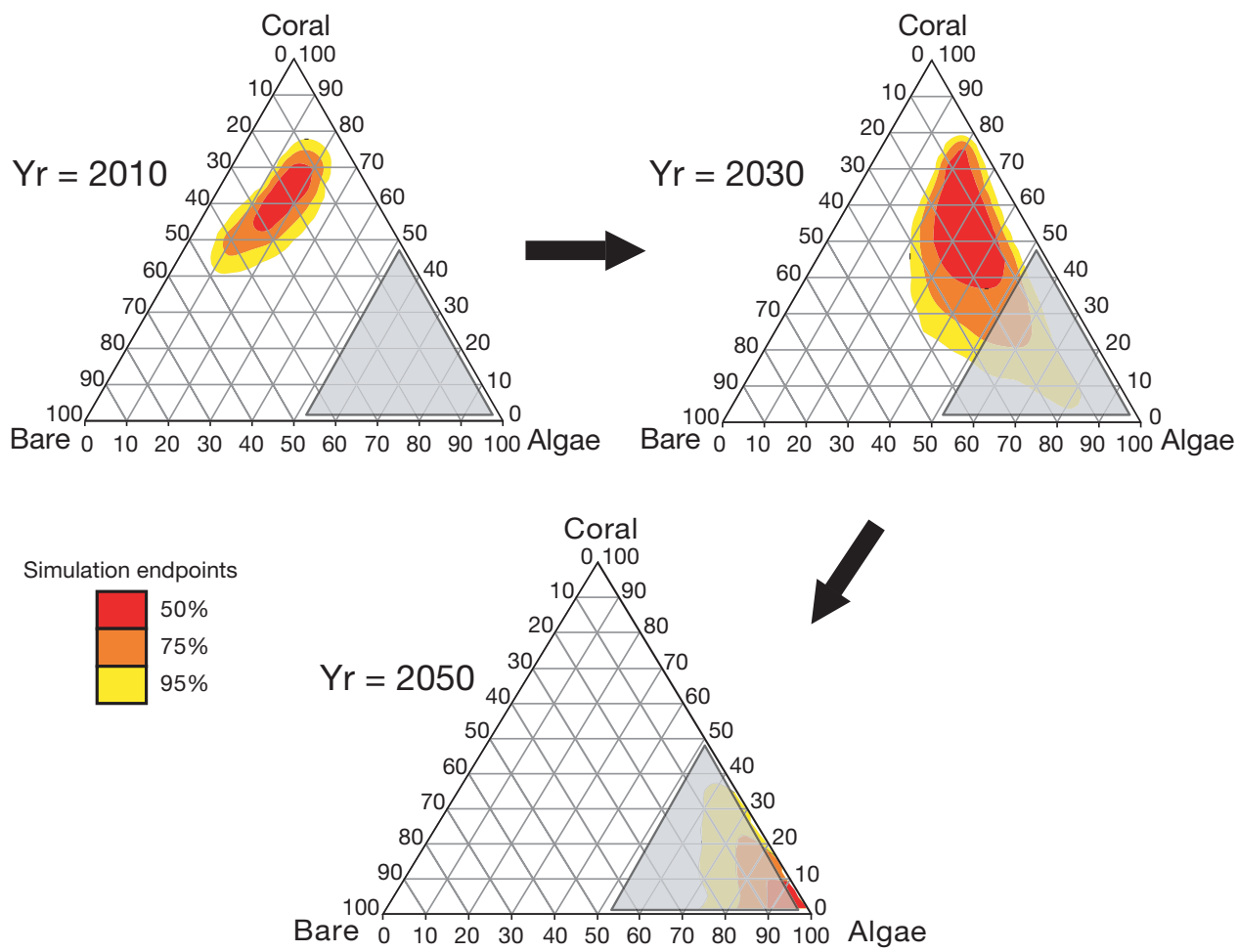

Fig. 5. Sample ReefState simulation, demonstrating presentation of coral-algae-bare output densities (50, 75 and $95 \%$ ) on a ternary axis. Scenario reflects uncertainty surrounding temporal progression from a coral-dominated reef state in 2010 towards an algaldominated state in 2050. Proportion of simulation endpoints falling within shaded zone represents $p$ (algae cover $>50 \%$ ). Within the ternary space, any coral-algae-bare zone of interest can be similarly interrogated to determine its likelihood of occurrence 
when we enforce a high constraint to macroalgae growth, then the same level of confidence of retaining a hard coral dominated reefscapes is extended at least 20 yr longer than for the low constraint simulations (Fig. 6a). Similar trends are observed for the high warming scenario (Fig. 6b). This result has important implications for coral reef managers, suggesting that local measures to contain the proliferation of macroalgae may be rewarded in terms of marginal improvement in hard coral resilience, despite climate warming. Irrespective of algal constraints however, as water temperatures exceed the tolerance of even the most heat resistant coral species, the likelihood of retaining hard coral dominance is diminished.

\section{Shifts in coral composition}

There is potential for shifts in the dominant hard coral genus that reflect both their different sensitivities to extended high water temperatures and their alternative recovery traits. In Fig. 7 these are represented as probability plots describing the likelihood for each of our indicator hard coral genera being less than their initial 1990 levels (Fig. 7). For both the low (Fig. 7a) and high (Fig. 7b) warming scenarios, the long-term
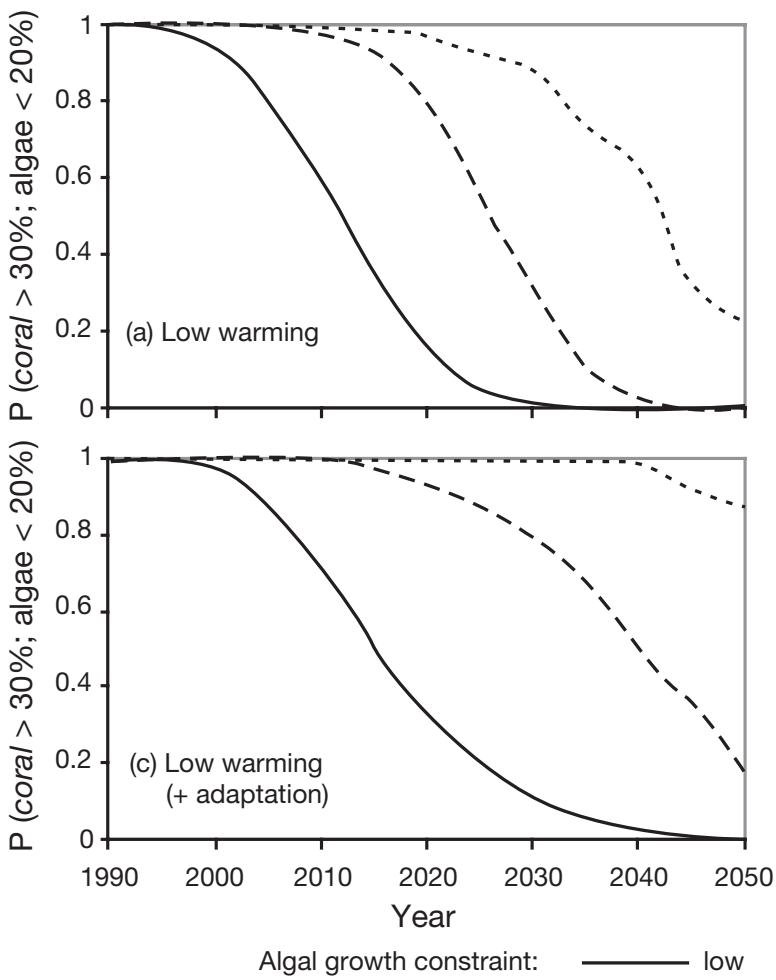

Fig. 6. Probability plots of likelihood of future reefscapes being coral-dominated (i.e. combined Acropora spp. and Porites spp. cover $>30 \%$; algae cover $<20 \%$ ) for alternate combinations of warming scenario and low, medium and high algal growth constraint. (a) Low warming and (b) high warming scenarios; (c,d) implication of $+0.1^{\circ} \mathrm{C}$ decade ${ }^{-1}$ adaptation in thermal bleaching threshold for (c) low warming and (d) high warming scenarios survival strategy of Porites spp. (heat tolerant [see Fig. A3b], slow recovery [see Fig. A5b]) appears to be superior to that of Acropora spp. (heat sensitive [see Fig. A3a], fast recovery [see Fig. A5a]). However, there is an interim phase until 2020, which is characterised by low intensity events and longer recurrence intervals, where the Acropora spp. survival strategy is superior due to its faster rate of return from relatively low mortality levels. A clear transition point, however, is evident around 2020, as bleaching events with durations of 60 to $80 \mathrm{~d}$ become a potential reality and the average recurrence interval between events is reduced to 2-3 yr (see Fig. A1a).

\section{Effect of adaptation}

The effect of $\mathrm{a}+0.1^{\circ} \mathrm{C}$ decade $^{-1}$ adaptation in the thermal bleaching threshold (see Fig. A1b) is to delay the arrival of the transition point until around 2040 for the low warming scenario (Fig. 7c) and 2030 for the reef-building states, adaptation of $+0.1^{\circ} \mathrm{C}$ decade $^{-1}$ would not be sufficient to prevent Porites spp.-type reefscapes becoming more commonplace than Acropora spp.-type reefscapes by 2050 .
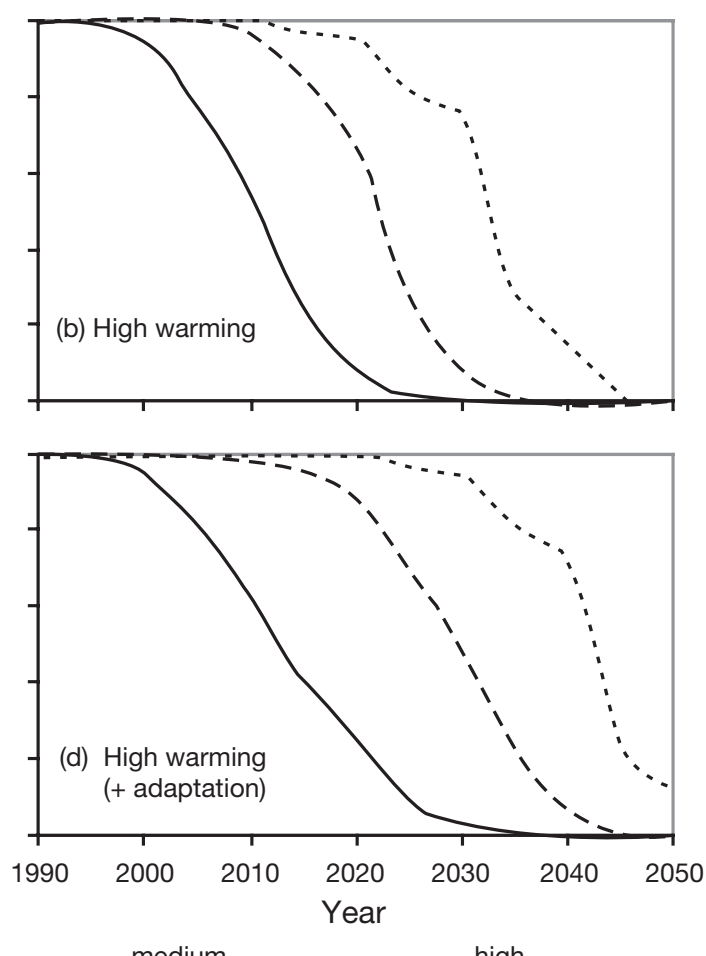

- - - - medium -...... high high warming scenario (Fig. $7 \mathrm{~d}$ ). In terms of future 

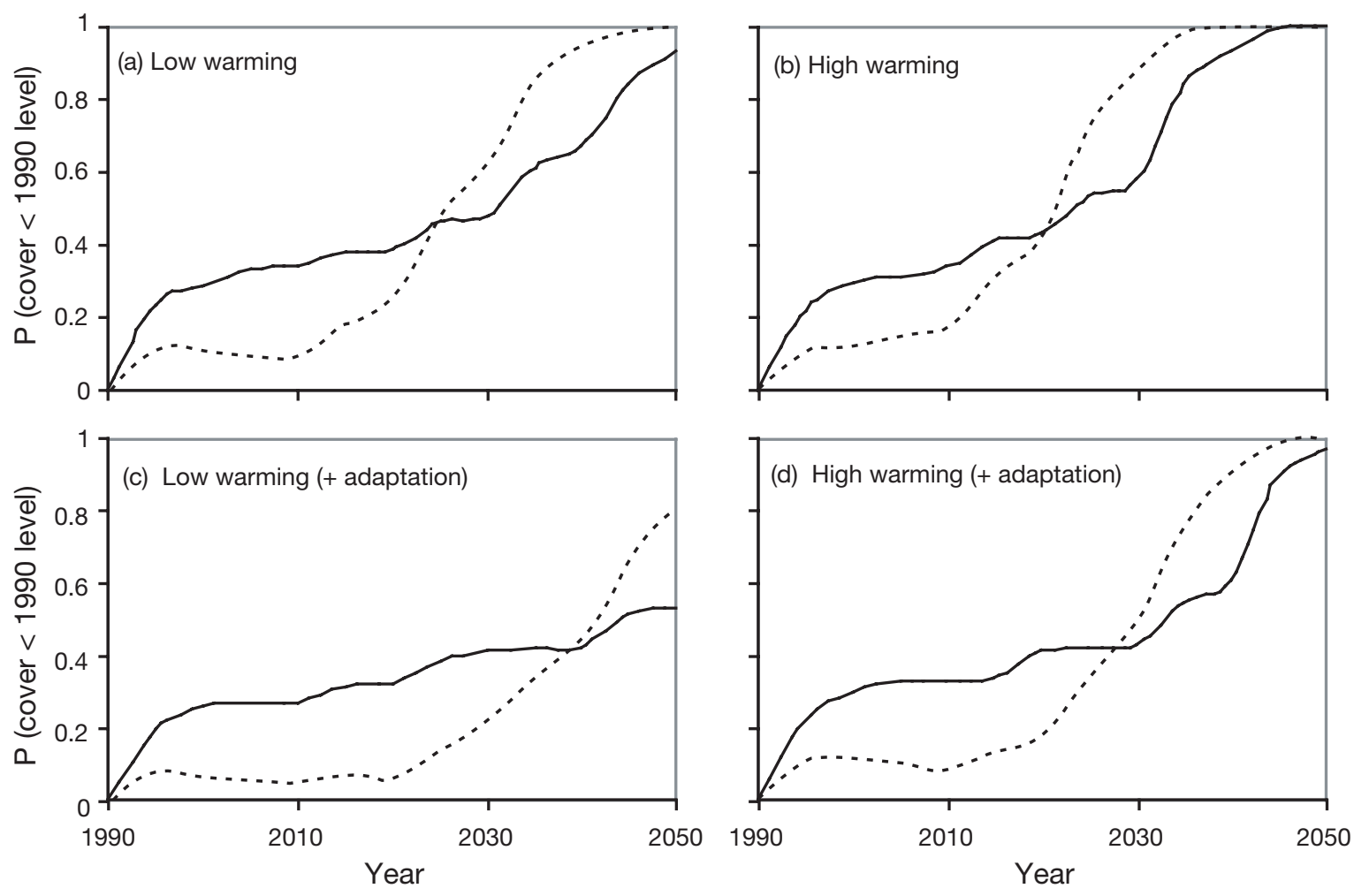

Porites spp.

Acropora spp.

Fig. 7. Probability plots of likelihood of Acropora spp. cover (fast growing, heat sensitive) and Porites spp. cover (slow growing, heat tolerant) being less than their 1990 levels. (a) Low warming and (b) high warming scenarios; $(\mathrm{c}, \mathrm{d})$ implication of $+0.1^{\circ} \mathrm{C}$ decade $^{-1}$ adaptation in thermal bleaching threshold for (c) low warming and (d) high warming scenarios

\section{Synergistic effects of adaptation and constraint on algal growth}

The potential benefit to be gained from a $+0.1^{\circ} \mathrm{C}$ decade $^{-1}$ increase in thermal tolerance is also strongly enhanced by higher constraints on algal growth (Fig. 6c,d). With low constraint, only modest benefits are achieved ( 10 year difference for equivalent probability confidence levels) in terms of ensuring the maintenance of hard coral dominated reefscapes. The result highlights that the potential for recovery of damaged coral communities through reseeding from more resistant populations (Done et al. 2003, Coles \& Brown 2004) is heavily reliant upon the existence of recovery conditions that ensure reef substrate is kept free of carpets of algae, to assist the growth of remnant corals and survival and growth of larval recruits.

\section{Likelihood of algal dominance}

Not surprisingly, the likelihood of algae contributing more than $50 \%$ of the total bottom cover is signifi- cantly impacted by the level of algal constraint applied in the model (Fig. 8a,b). For example, the likelihood distribution is virtually the same for low warming with a low algal growth constraint scenario and high warming with medium algal constraint. Again the years between 2020 and 2030 see the complete loss of the buffering capacity to absorb increasingly intense and frequent bleaching impacts. The $+0.1^{\circ} \mathrm{C}$ decade $^{-1}$ adaptation in thermal bleaching threshold (Fig. 8c,d) extends the buffering capacity phase by 1 to 2 decades.

For high algal constraint levels, macro algal cover is constrained, a priori, to its 1990 level of $10 \%$. Notwithstanding this release from competition with macroalgae, corals do not retain dominance beyond 2030 . Rather, reduced return intervals between worsening coral mortality events result in a non-reef building state with large amounts of substrate occupied by neither corals nor macroalgae. In management terms, such a reef state may or may not have improved chances for successful settlement and establishment of reef-building taxa, should a viable recruitment source be available (Done 1992, McCook 1999, McClanahan et al. 2003). 

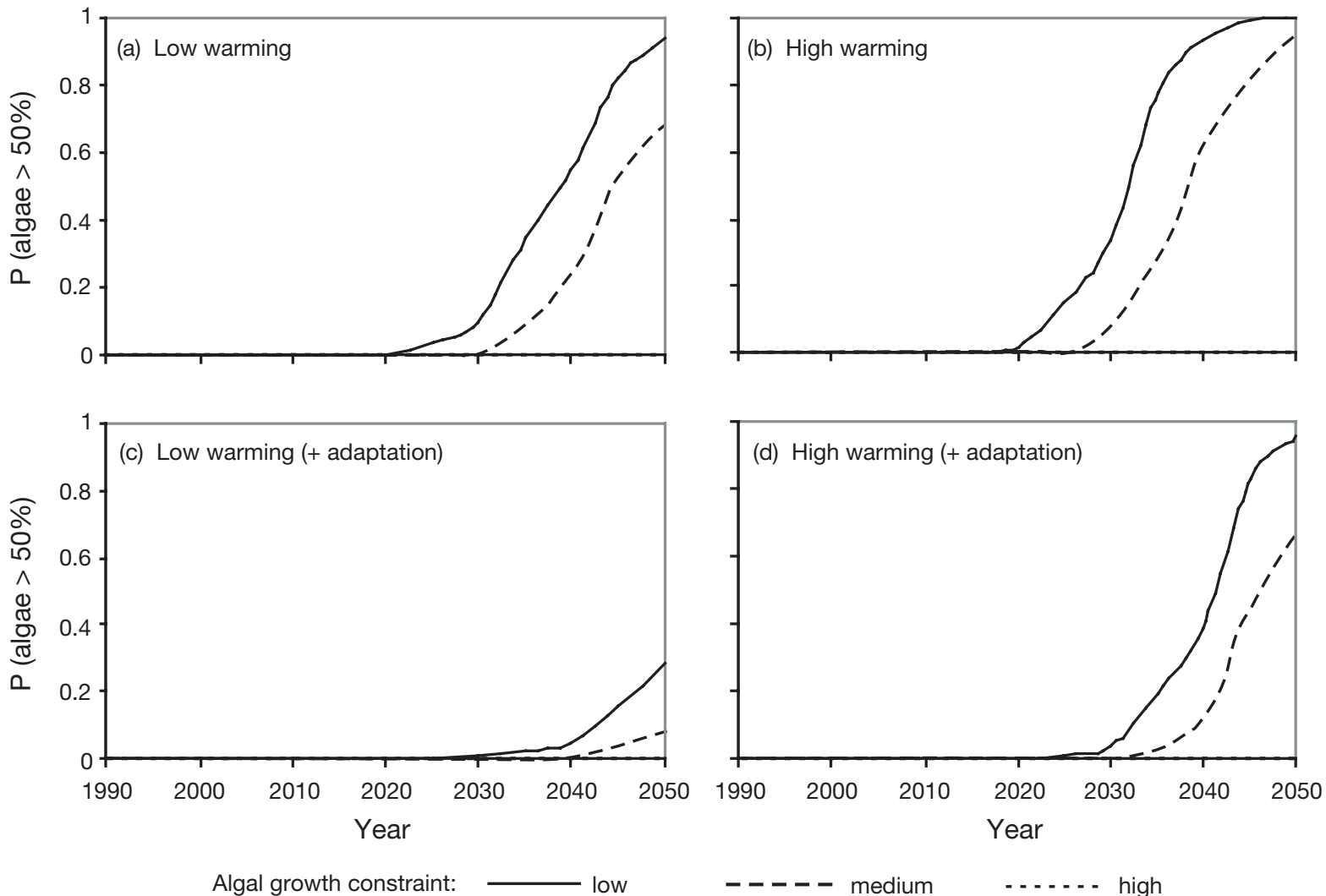

Fig. 8. Probability plots of likelihood of future reefscapes being algae-dominated (i.e. algae contributing $>50 \%$ of total cover) for alternate combinations of warming scenario and low, medium and high algal growth constraint. (a) Low warming and (b) high warming scenarios; $(\mathrm{c}, \mathrm{d})+0.1^{\circ} \mathrm{C}$ decade $^{-1}$ adaptation in thermal bleaching threshold for (c) low warming and (d) high warming scenarios

\section{Ecological lessons and implications for management}

The analysis suggests that there can be an improved outlook for coral dominance on inshore reefscapes, but only under conditions in which proliferation of prostrate macroalgae is subject to external constraints. This study therefore highlights the importance of control of macroalgae as a goal for management. Existing and proposed measures to reduce nutrient pollution at site and regional scales of the GBR are clearly pertinent, and measures to increase herbivory, or otherwise control local algal growth, also warrant consideration. Above certain thresholds of herbivore abundance, consumption can track and absorb substantial increases in algal production (Russ \& McCook 1999). This particular non-linear relationship between algal biomass change and herbivore population size suggests that near the threshold, small increases in herbivore population size could lead to significant reductions in algal biomass accumulation. The key scientific questions thus relate to the critical threshold for herbivore abundance and the extent to which controllable factors (e.g. level of fishing pressure, habitat complexity, water quality parameters) may need to be altered in order to restore herbivore populations towards this threshold. The modular and adaptive nature of the modelling approach and framework described here provides the basis for future exploration of such issues and broader management issues in complex environmental and socio-economic contexts.

In all but a few best-case scenarios, the bleaching disturbance regime modified successional trajectories, as more heat-tolerant species (e.g. most species of massive Porites spp.) are differentially favoured at the expense of heat-sensitive species (e.g. most species of Acropora spp.). Should macroalgae expand at near maximum rates into space made available following bleaching-induced mortality, then it can competitively pre-empt space and exclude all hard corals by 2040 . But should it be constrained naturally or by management intervention, then the probability of a viable coral population extending beyond 2050 is increased. Irrespective of algal constraints however, should water temperatures exceed the tolerance of even the most heat resistant coral species, reefs will be quickly transformed into unattractive, unproductive remains of a reef devoid of significant coral cover and associated biodiversity. 
For our best case scenarios, in which we assumed there would be an increase in thermal tolerance of both types of corals at a rate of $+0.1^{\circ} \mathrm{C}$ decade $^{-1}$, we projected a considerable delay (10 to $20 \mathrm{yr}$ ) in decline under both the low and high warming scenarios. More heat resistant strains of zooxanthellae do exist (e.g. Baker et al. 2004, Little et al. 2004, Rowan 2004), but the speed and extent to which they can proliferate and thereby produce more heat-adapted coral populations and communities is currently unknown. What is clear, however, is that the successful establishment of more resistant coral populations from newly settled spat or small remnants is heavily reliant upon the existence of recovery conditions at damaged sites that ensure the substrate is kept free of algal carpets. On the Great Barrier Reef, resilience-based management is being implemented through major initiatives including the expansion of no-take reserves, improved fisheries management and a programme to halt and reverse inputs of land-based sources of pollution. Further research and monitoring is necessary to increase our knowledge about critical sources of resilience, and to better define the nature and practicality of effective management interventions.

In summary, the results of our most basic simulations to the year 2050 are consistent with those of previous studies (e.g. Hoegh-Guldberg 1999, Sheppard 2003) in demonstrating the potential for unprecedented levels of future coral reef decline given even modest scenarios for future climate warming. However, simulations that take into account ecological and adaptive processes suggest that there may be capacity for coral communities to buffer these negative declines. Potentially effective mechanisms of acclimatization of individual corals are known, but the capacity and likely rates of similar transformations for entire populations and communities that define coral reefscapes cannot even be guessed at. Notwithstanding this uncertainty, it is clear that management for good on-site conditions greatly enhances the ecological benefits gained from marginal increases in coral survivorship. Those regional to local factors that are the focus of management attention on coral reefs (viz. water quality, fishing pressure, and no take zones) could in principle contribute to the restoration of local biodiversity and ecosystem function (Done et al. 1996). Future extensions are planned for the ReefState model that will help us explore these ideas further.

\section{LITERATURE CITED}

Allen MR, Stott PA, Mitchell JFB, Schnur R, Delworth TL (2000) Quantifying the uncertainty in forecasts of anthropogenic climate change. Nature 407:617-620

Baker AC, Starger CJ, McClanahan TR, Glynn PW (2004)
Corals' adaptive response to climate change. Nature 430 : 741

Bell PRF (1992) Eutrophication and coral reefs - some examples in the Great Barrier Reef lagoon. Water Res 26: $553-568$

Bellwood DR, Hughes TP, Folke C, Nyström M (2004) Confronting the coral reef crisis. Nature 429:827-833

Berkelmans R (2002) Time-integrated thermal bleaching thresholds of reefs and their variation on the Great Barrier Reef. Mar Ecol Prog Ser 229:73-82

Berkelmans R, De'ath G, Kininmonth S, Skirving WJ (2004) A comparison of the 1998 and 2002 coral bleaching events on the Great Barrier Reef: spatial correlation, patterns and predictions. Coral Reefs 23:74-83

Borsuk ME, Stow CA, Reckhow KH (2004) A Bayesian network of eutophication models for synthesis, prediction, and uncertainty analysis. Ecol Model 173:219-239

Brodie J, Furnas MJ (1996) Cyclones, river flood plumes and natural water quality extremes in the central Great Barrier Reef. In: Hunter HM, Eyles AG, Rayment GE (eds) Downstream effects of land use. Department of Natural Resources, Brisbane, p 367-374

Buddemeier RW, Kleypas JA, and Aronson RB (2004) Coral reefs and global climate change: potential contributions of climate change to stresses on coral reef ecosystems. Pew Center on Global Climate Change, Arlington, VA

Clarke JS, Carpenter SR, Barber M, Collins S and 11 others (2001) Ecological forecasts: an emerging imperative. Science 293:657-660

Coles SL, Brown BE (2004) Coral bleaching. Capacity for acclimatization and adaptation. Adv Mar Biol 46:183-223

Diaz-Pulido G, McCook LJ (2002) The fate of bleached corals: patterns and dynamics of algal recruitment. Mar Ecol Prog Ser 232:115-128

Diaz-Pulido G, McCook LJ (2003) Relative roles of herbivory and nutrients in the recruitment of coral-reef seaweeds. Ecology 84:2026-2033

Diaz-Pulido G, McCook LJ (2004) Effects of live coral, epilithic algal communities and substrate type on algal recruitment. Coral Reefs 23:225-233

Done TJ (1992) Phase shifts in coral reef communities and their ecological significance. Hydrobiologia 247:121-132

Done TJ (1999) Coral community adaptability to environmental change at the scales of regions, reefs and reef zones. Am Zool 39:66-79

Done TJ, Ogden JC, Wiebe WJ, Rosen BR (1996). Biodiversity and ecosystem function of coral reefs. In: Mooney HA, Cushman JH, Medina E, Sala OE, Schultze ED (eds) Functional roles of biodiversity: a global perspective. John Wiley, Chichester, p 393-429

Done TJ, Whetton P, Jones R, Berkelmans R, Lough J, Skirving W, Wooldridge S (2003) Global climate change and coral bleaching on the Great Barrier Reef. Department of Natural Resources and Mines, Queensland, Australia (also available at www.nrm.qld.gov.au/science/pdf/ barrier_reef_report_1.pdf)

Gelman A, Carlin JB, Stern HS, Rubin DB (1997) Bayesian data analysis. Chapman \& Hall, London

Hoegh-Guldberg O (1999) Climate change, coral bleaching and the future of the world's coral reefs. Mar Freshw Res 50:839-866

Hoegh-Guldberg H, Hoegh-Guldberg O (2004) The implications of climate change for Australia's Great Barrier Reef. People and industries at risk. World Wildlife Fund and Queensland Tourism Industry Council, Sydney

Hosmer DW, Lemeshow S (1999) Applied survival analysis. Wiley \& Sons, New York 
Hubbell SP (1997) A unified theory of biogeography and relative species abundance and its application to tropical rain forests and coral reefs. Coral Reefs 16:S9-S21

Hughes TP, Baird AH, Dinsdale EA, Moltschaniwskyj NA, Pratchett MS, Tanner JE, Willis BL (1999) Patterns of recruitment and abundance of corals along the Great Barrier Reef. Nature 397:59-63

Hughes TP, Baird AH, Bellwood DR, Card M and 13 others (2003) Climate change, human impacts, and the resilience of coral reefs. Science 301:929-933

IPCC (Intergovernmental Panel on Climate Change) (2001) Climate change 2001: synthesis report. A Contribution of Working Groups I, II, and III to the Third Assessment Report of the Intergovernmental Panel on Climate Change. Cambridge University Press, Cambridge

Kerr RA (1997) Climate change: greenhouse forecasting still cloudy. Science 276:1040-1042

Kinzig A, Starrett D (2003) Coping with uncertainty: a call for a new science-policy forum. Ambio 32:330-335

Klumpp DW, McKinnon AD (1992) Community structure, biomass and productivity of epilithic algal communities on the Great Barrier Reef: dynamics at different spatial scales. Mar Ecol Prog Ser 86:77-89

Klumpp DW, McCook LJ, Done TJ, McKinnon AD (1999) Nutrient enhancement of nearshore reefs: baseline information of nutrient concentrations, primary production and reef community structure. Great Barrier Reef Marine Park Authority, Townsville

Kuczera G (1997) Efficient subspace probabilistic parameter optimisation for catchment models. Water Resour Res 33:177-185

Kuczera G, Parent E (1998) Monte Carlo assessment of parameter uncertainty in conceptual catchment models: the metropolis algorithm. J Hydrol (Aust) 211:69-85

Little AF, van Oppen MJH, Willis BL (2004) Flexibility in algal endosymbioses shapes growth in reef corals. Science 304:1492-1494

Loya Y, Sakai K, Yamazoto K, Nakano Y, Sambali H, van Woesik R (2001) Coral bleaching: the winners and losers. Ecol Lett 4:122-131

Marshall PA, Baird AH (2000) Bleaching of corals in the Central Great Barrier Reef: variation in assemblage response and taxa susceptibilities. Coral Reefs 19:155-163

McClanahan TR, Sala E, Stickels P, Cokos BA, Baker A, Starger CJ (2003) Interaction between nutrients and herbivory in controlling algal communities and coral condition in Glovers Reef, Belize. Mar Ecol Prog Ser 261: $135-147$

McCook LJ (1996) Effects of herbivores and water quality on the distribution of Sargassum on the central Great Barrier Reef: cross-shelf transplants. Mar Ecol Prog Ser 139: 179-192

McCook LJ (1999) Macroalgae, nutrients and phase shifts on coral reefs: scientific issues and management consequences for the Great Barrier Reef. Coral Reefs 18:357-367

McCook LJ (2001) Competition between corals and algal turfs

Editorial responsibility: Otto Kinne (Editor-in-Chief), Oldendorf/Luhe, Germany along a gradient of terrestrial influence in the nearshore central Great Barrier Reef. Coral Reefs 19:419-425

Neapolitan RE (1990) Probabilistic reasoning in expert systems: theory and algorithms. John Wiley \& Sons, New York

Ninio R, Meekan M, Done T, Sweatman H (2000) Temporal patterns in coral assemblages on the Great Barrier Reef from local to large spatial scales. Mar Ecol Prog Ser 194: $75-74$

Pandolfi JM (1999) Response of Pleistocene coral reefs to environmental change over long temporal scales. Am Zool 39:113-130

Pearl J (1986) Fusion, propagation, and structuring in belief networks. Artif Intelligence 29:241-288

Pearl J (1988) Probabilistic reasoning in intelligent systems: networks of plausible inference. Morgan Kaufmann, San Mateo, CA

Pearl J (1999) Graphs, structural models, and causality. In: Glymour C, Cooper GF (eds) Computation, causation and discovery. AAAI Press, Menlo Park, CA, p 95-138

Reckhow KH (1999) Water quality prediction and probability network models. Can J Fish Aquat Sci 56:1150-1158

Rowan R (2004) Thermal adaptation in reef coral symbionts. Nature 430:742

Russ GR, McCook LJ (1999) Potential effects of a cyclone on benthic algal production and yield to grazers on coral reefs across the central Great Barrier Reef. J Exp Mar Biol Ecol 235:237-254

Scott FJ, Russ GR (1987) Effects of grazing on species composition of the epilithic algal community on coral reefs of the central Great Barrier Reef. Mar Ecol Prog Ser 39:293-304

Semenov MA, Brooks RJ, Barrow EM, Richardson CW (1998) Comparison of the WGEN and LARS-WG stochastic weather generators for diverse climates. Clim Res 10: 95-107

Sheppard CRC (2003) Predicted recurrences of mass coral mortality in the Indian Ocean. Nature 425:294-297

van Woesik R (2002) Processes regulating coral communities. Comments Theor Biol 7:201-214

van Woesik R, Tomascik T, Blake S (1999) Coral assemblages and physico-chemical characteristics of the Whitsunday Islands: evidence of recent community changes. Mar Freshw Res 50:427-440

West JM, Salm RV (2003) Resistance and resilience to coral bleaching: Implications for coral reef conservation and management. Conserv Biol 17:956-967

Wilkinson C (2004) Status of coral reefs of the world. Australian Institute of Marine Science, Townsville

Williams DMcB (1991) Patterns and processes in the distribution of coral reef fishes. In: Sale PF (ed) The ecology of fishes on coral reefs. Academic Press, San Diego, CA, p 437-474

Wooldridge SA, Done TJ (2004) Learning to predict largescale coral bleaching from past events: a Bayesian approach using remotely sensed data, in-situ data, and environmental proxies. Coral Reefs 23:96-108

Submitted: October 18, 2004; Accepted: January 10, 2005

Proofs received from author(s): May 30, 2005 